\title{
Ultraconserved elements (UCEs) illuminate the population genomics of a recent, high-latitude avian speciation event
}

\author{
Kevin Winker ${ }^{\text {Corresp., }}{ }^{1}$, Travis C Glenn ${ }^{2}$, Brant C Faircloth ${ }^{3}$ \\ 1 University of Alaska Museum \& Department of Biology and Wildlife, University of Alaska Fairbanks, Fairbanks, Alaska, USA \\ 2 Department of Environmental Health Science and Institute of Bioinformatics, University of Georgia, Athens, United States \\ 3 Department of Biological Sciences and Museum of Natural Science, Louisiana State University, Louisiana State University, Baton Rouge, USA \\ Corresponding Author: Kevin Winker \\ Email address: kevin.winker@alaska.edu
}

Using a large, consistent set of loci shared by descent (orthologous) to study relationships among taxa would revolutionize among-lineage comparisons of divergence and speciation processes. Ultraconserved elements (UCEs), highly conserved regions of the genome, offer such genomic markers. The utility of UCEs for deep phylogenetics is clearly established and there are mature analytical frameworks available, but fewer studies apply UCEs to recent evolutionary events, creating a need for additional example datasets and analytical approaches. We used UCEs to study population genomics in snow and McKay's buntings (Plectrophenax nivalis and $P$. hyperboreus). Prior work suggested divergence of these sister species during the last glacial maximum ( 18-74 Kya). With a sequencing depth of $\sim 30 \times$ from four individuals of each species, we used a series of analysis tools to genotype both alleles, obtaining a complete dataset of 2,635 variable loci ( 3.6 single nucleotide polymorphisms [SNPs]/locus) and 796 invariable loci. We found no fixed allelic differences between the lineages, and few loci had large allele frequency differences. Nevertheless, individuals were $100 \%$ diagnosable to species, and the two taxa were different genetically $\left(F_{S T}=0.034 ; P=0.03\right)$. The demographic model best fitting the data was one of divergence with gene flow. Estimates of demographic parameters differed from published mtDNA research, with UCE data suggesting lower effective population sizes ( 92,500 240,500 individuals), a deeper divergence time ( $241,000 \mathrm{yrs}$ ), and lower gene flow (2.85.2 individuals per generation). Our methods provide a framework for future population studies using UCEs, and our results provide additional evidence that UCEs are useful for answering questions at shallow evolutionary depths. 
1 Ultraconserved elements (UCEs) illuminate the population genomics of a recent, high-

2 latitude avian speciation event

3

\section{Kevin Winker ${ }^{1}$, Travis C. Glenn ${ }^{2}$, and Brant C. Faircloth ${ }^{3}$}

${ }^{1}$ University of Alaska Museum, 907 Yukon Drive, Fairbanks, AK 99775, USA

2 Department of Environmental Health Science and Institute of Bioinformatics, University of Georgia, Athens, GA 30602, USA

${ }^{3}$ Department of Biological Sciences and Museum of Natural Science, Louisiana State University, Baton Rouge, LA 70803, USA

Corresponding author: Kevin Winker, University of Alaska Museum, 907 Yukon Drive, Fairbanks, AK 99775, USA; kevin.winker@alaska.edu; 907-474-7027; fax 907-474-5469

Running title: Bunting speciation using UCEs

Using a large, consistent set of loci shared by descent (orthologous) to study relationships among taxa would revolutionize among-lineage comparisons of divergence and speciation processes. Ultraconserved elements (UCEs), highly conserved regions of the genome, offer such genomic markers. The utility of UCEs for deep phylogenetics is clearly established and there are mature analytical frameworks available, but fewer studies apply UCEs to recent evolutionary events, creating a need for additional example datasets and analytical approaches. We used UCEs to study population genomics in snow and McKay's buntings (Plectrophenax nivalis and $P$. hyperboreus). Prior work suggested divergence of these sister species during the last glacial maximum ( 18-74 Kya). With a sequencing depth of $\sim 30 \times$ from four individuals of each species, we used a series of analysis tools to genotype both alleles, obtaining a complete dataset of 2,635 variable loci ( 3.6 single nucleotide polymorphisms [SNPs]/locus) and 796 invariable loci. We found no fixed allelic differences between the lineages, and few loci had large allele frequency differences. Nevertheless, individuals were $100 \%$ diagnosable to species, and the two taxa were different genetically $\left(F_{S T}=0.034 ; P=0.03\right)$. The demographic model best fitting the data was one of divergence with gene flow. Estimates of demographic parameters differed from published mtDNA research, with UCE data suggesting lower effective population sizes $(\sim 92,500-240,500$ individuals), a deeper divergence time ( 241,000 yrs), and lower gene flow (2.8-5.2 individuals per generation). Our methods provide a framework for future population studies using UCEs, 
34 and our results provide additional evidence that UCEs are useful for answering questions at 35 shallow evolutionary depths.

\section{Introduction}

Among non-model organisms, population genetic studies have used a diverse set of markers, tending to concentrate on those with sufficiently high substitution rates to provide useful data at shallow levels of evolutionary divergence, e.g., from the populations-to-species levels (Avise 1994, Hillis et al. 1996, Pearse \& Crandall 2003). This approach usually provides answers to the specific questions asked by researchers, but the historic focus on markers with high substitution rates has produced studies that include relatively few loci and often have little to no overlap with loci used for other taxa. This lack of consistency in the loci used across different studies compromises our ability to make direct comparisons of population genetic parameters among taxa (e.g., in divergence statistics and in estimates of gene flow and effective population sizes). Improvements in sequencing platforms and genomic data collection approaches are changing this general pattern by enabling us to efficiently collect much larger samples of the genome, up to and including whole-genome sequences (Ellegren 2014). However, the sheer quantity of data obtained from whole-genome sequencing can require excessively long computation times, and may be overkill for many questions. The parallel difficulties of collecting a moderate sample of the genome from identical loci across diverse species argue for a sequence data collection approach that a) subsamples the genome to b) obtain orthologous markers across a broad taxonomic scope. This type of approach would provide a tractable number of loci for analyses while improving among-study comparisons and larger-scale comparative metaanalyses. Ultraconserved elements (UCEs) are one class of genome-wide marker that might provide a solution to these problems.

UCEs are conserved sequences shared among divergent animal genomes (Bejerano et al. 2004, Siepel et al. 2005, Stephen et al. 2008, Janes et al. 2011), and many UCE loci are likely to be involved in controlling gene expression (Marcovitz et al. 2016). UCEs in vertebrates show

61 little overlap with most types of paralogous genes, and, as a marker class, UCE loci are broadly

62 distributed across the genome and are typically transposon-free (Derti et al. 2006, Simons et al.

63 2006, McCormack et al. 2011, Harvey et al. 2016). We focus on the set of UCEs previously

64 defined for tetrapods and now in widespread use (McCormack et al. 2011, Faircloth et al. 2012). 
65 Outside of their functional relevance, UCE loci have demonstrated utility for recovering deeper66 level phylogenetic relationships (McCormack et al. 2013, Faircloth et al. 2015, Gilbert et al.

67 2015) and shallower-level genus and population relationships (Smith et al. 2014, Harvey \& Brumfield 2015, Leaché et al. 2015, Harvey et al. 2016, Manthey et al. 2016, Oswald et al. 2016, Mason et al. 2018). Although UCEs are highly conserved at their core, which enables universal capture of loci across diverse groups of organisms (Faircloth et al. 2012, 2013, 2015; Starret et al. 2016), lower levels of purifying selection away from the core allow substitutions to accumulate in the flanking regions. Using human genome data, Faircloth et al. (2012) demonstrated that the increased variation in UCE flanking sequence might be adequate to make these loci useful for questions at shallow levels of divergence, and this hypothesis has been supported by subsequent empirical studies (Smith et al. 2014, Harvey \& Brumfield 2015, Harvey et al. 2016, Oswald et al. 2016, Mason et al. 2018). However, the utility of UCE loci for studying population genetics, population divergence, and/or incipient speciation is only beginning to be tested, and both the value and challenges of using UCEs at these shallow levels remain underexplored.

Here, we examine the utility of UCEs for studying the population genomics of divergence between two bird species, McKay's bunting (Plectrophenax hyperboreus) and snow bunting ( $P$. nivalis). McKay's buntings breed on remote islands in the Bering Sea (where our samples are from) and are the highest-latitude endemic songbirds; their range is restricted to the North Pacific region. Snow buntings breed throughout the rest of the high-latitude Holarctic (our samples are from the southern edge of the Being sea on the Alaska Peninsula and an Aleutian island; Table S1). McKay's bunting is thought to have arisen $~ 18-74$ Kya during the last glacial maximum (LGM) through divergence from snow buntings, and previous work suggests gene flow between the two may be ongoing (Maley \& Winker 2010). These species are interesting to study using UCEs because prior work (Maley \& Winker 2010) enables us to compare population genetic statistics derived from UCEs versus traditional population genetic markers (mtDNA sequence and amplified fragment length polymorphisms, AFLPs). These species also allow us to test the utility of UCEs for studying very shallow divergences between sister lineages where gene flow may be ongoing.

\section{Methods}


Laboratory.-We extracted DNA from muscle tissue of eight specimens (four of each species) studied by Maley \& Winker (2010) using proteinase K digestion (100 mM Tris pH 8, 50 $\mathrm{mM}$ EDTA, $0.5 \% \mathrm{SDS}, 1 \mathrm{mg} / \mathrm{mL}$ proteinase K) followed by SPRI bead purification (Rohland \& Reich 2012); Supplemental Information, Table S1). We chose this sample size (and our sequencing depth) to ensure that we could confidently call both alleles for each individual in each population to achieve eight sequences per population at each locus, which Felsenstein (2005) considered to be the optimum sample size for coalescent-based analyses. Following DNA extraction, we prepared dual-indexed DNA libraries for each sample using methods described in Glenn et al. (2017). After library preparation, we quantified each library using a Qubit fluorimeter (Invitrogen, Inc.), and we combined eight libraries into equimolar pools of $500 \mathrm{ng}$ each (62.5 ng/library). We enriched the pool of 8 samples for 5,060 UCE loci using the Tetrapods-UCE-5Kv1 kit from MYcroarray following version 1.5 of the UCE enrichment protocol and version 2.4 of the post-enrichment amplification protocol (ultraconserved.org) with HiFi HotStart polymerase (Kapa Biosystems) and 14 cycles of post-enrichment PCR. We then quantified the fragment size distribution of the enriched pool on a Bioanalyzer (Agilent, Inc.) and qPCR quantified the enriched pool using a commercial kit (Kapa Biosystems). We combined the enriched pool of eight bunting samples with enriched pools from other birds at equimolar ratios, and we sequenced the resulting pool using one lane of paired-end $150 \mathrm{bp}$ (PE150) sequencing on an Illumina HiSeq 2500 (UCLA Neuroscience Genomics Core).

Bioinformatics.-After sequencing, we demultiplexed the sequencing reads using bcl2fastq version 1.8.4 (Illumina, Inc.), and we trimmed the demultiplexed reads for adapter contamination and low-quality bases using a parallel wrapper (Faircloth 2013) around

118 Trimmomatic (ver. 0.32 Bolger et al. 2014). We then combined singleton reads that lost their mate with read 1 files, combined all individual read 1 files (plus singletons) together and all individual read 2 files together, and assembled these two read 1 and read 2 files de novo using

121 Trinity (ver. 2.0.6; Grabherr et al. 2011) on Galaxy (Afgan et al. 2016). After assembling this 122 composite of data from all individuals, we used Phyluce (ver. 1.4.0; Faircloth 2016) to identify

123 FASTA sequences from orthologous UCEs and remove FASTA sequences from non-UCE loci 124 or potential paralogs. We called the resulting file our reference set of UCE loci, which we used 125 as the reference sequence for calling individual variants. 
Next, we used Phyluce and its program dependencies (BWA 0.7.7, Li \& Durbin 2009;

\section{7}

128

129

130

131

132

133

134

135

136

137

138

139

140

141

142

143

144

145

146

147

148

149

150

151

152

153

154

155

156

SAMtools 0.1.19, Li et al. 2009; Picard 1.106, http://broadinstitute.github.io/picard) to align unassembled, raw reads from individual buntings to the reference set of UCE loci. Specifically, this workflow aligned raw reads on a sample-by-sample basis against the composite reference using the bwa-mem algorithm (preferred for reads $>70 \mathrm{bp}$; Li 2013); added header information to identify alignments from individual samples; cleaned, validated, and marked duplicates in the resulting BAM (Binary Alignment/Map) file using Picard; and merged all individuals into a single BAM file using Picard. Following preparation of the merged BAM, we used GATK (ver. 3.4-0; McKenna et al. 2010) to identify and realign indels, call and annotate single nucleotide polymorphisms (SNPs) and indels, and mask SNP calls around indels using a GATK workflow described as part of a population genomics pipeline for UCEs developed by Faircloth and Michael Harvey (https://github.com/mgharvey/seqcap_pop). This included restricting data to high-quality SNPs (Q30) and read-back phasing in GATK. After calling and annotating SNPs, we deviated from this workflow by using VCFtools (ver. 0.1.12b; Danacek et al. 2011) to filter the resulting variant call format (VCF) file with the --max-missing (1.0) and --minGQ (10.0) parameters, which created a complete data matrix with a minimum genotype quality (GQ) of 10 . We validated that GQ10 data were present for all individuals at all loci by visually assessing alignment data at 17 SNPs among 10 loci using Tablet (ver. 1.15.09.01; Milne et al. 2013). We used GATK's EMIT_ALL_CONFIDENT_SITES function to ensure that we only retained invariant loci with high quality (rather than missing) data. We then removed variable and invariable loci with incomplete data from downstream analyses, retaining only loci with complete data. This finalized our complete VCF file.

Data analysis.-We calculated coverage depths, SNP positions within loci, and SNPspecific and locus-specific $F_{S T}$ values on the complete VCF file using VCFtools (ver. 0.1.12b; Danacek et al. 2011). After thinning the VCF file to $1 \mathrm{SNP} /$ locus (which is required in demographic analyses when unlinked variation is important) and converting the VCF file to STRUCTURE format using PGDSpider (ver. 2.1.0.3; Lischer \& Excoffier 2012), we performed tests of Hardy-Weinberg equilibrium and computed observed and expected heterozygosities, homogeneity of variance, population structure (population $F_{S T}$, including a 10,000-replicate $G$ test; see Goudet et al. [1996]), and the probabilities of each individual's assignment to a particular population using Discriminant Analysis of Principal Components (DAPC) in adegenet 
157 (ver. 2.0.1; Jombart \& Ahmed 2011). To calculate nucleotide diversity, average distance between 158 taxa $\left(d_{\mathrm{xy}}\right)$, and net average distance $\left(d_{\mathrm{A}}\right)$, we created a concatenated FASTA file of all individual 159 sequences using catfasta2phyml by Johan Nylander

160 (https://github.com/nylander/catfasta2phyml), and we analyzed this file in MEGA (ver. 6;

161 Tamura et al. 2013) using the maximum composite likelihood method.

162 We estimated recombination using the four-gametes test as implemented in IMgc

163 (Woerner et al. 2007), which also produces sequence datasets from which the effects of 164 recombination have been removed. Resulting sequences were used for IMa2p (ver. 1.0,

165 Sethuraman and Hey 2015) analyses to attempt to estimate demographic parameters, but those 166 analyses did not converge under a variety of full- and sub-sampling schemes and are not 167 reported. We nevertheless include the results of IMgc because accounting for recombination is a critical part of workflows using full sequences (i.e., not just SNPs), and these results provide needed insight into the levels of recombination found in UCE loci for studies of this type.

We used Diffusion Approximations for Demographic Inference ( $\delta \mathrm{a} \delta \mathrm{i}$; ver. 1.7.0;

171 Gutenkunst et al. 2009) to infer demographic parameters from the data under a variety of

172 divergence scenarios (models) after excluding Z-linked loci (for $\delta a \delta i$ analyses only). Z-linked

173 loci in birds are on the sex chromosome, have a different inheritance scalar from autosomal loci,

174 and sample population sex ratios affect allele frequency estimates (e.g., Jorde et al. 2000,

175 Garrigan et al. 2007). We identified Z-linked loci in our data using BLASTn (ver. 2.3.1; Zhang

176 et al. 2000), by aligning the reference set of UCE loci against the zebra finch (Taeniopygia

177 guttata) genome (NCBI Annotation Release 103). We excluded UCE loci that strongly matched

178 ( $E$-values $\sim 0.0$ ) the zebra finch $Z$ chromosome. After removing $Z$-linked loci from our complete

179 VCF file, we converted this reduced dataset to biallelic format (which dropped one locus with $>2$

180 alleles at a SNP site) and thinned the data to one SNP per locus using VCFtools. Then we

181 converted the resulting VCF file to the joint site frequency spectrum (SFS) format required by

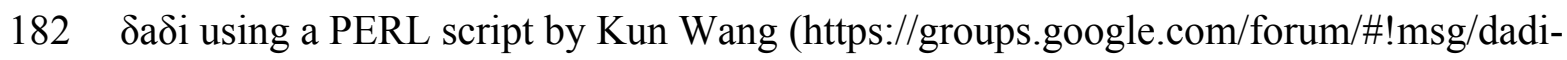

183 user/p1WvTKRI9_0/1yQtcKqamPcJ). Because we lacked an outgroup, we used a folded SFS in 184 our analyses (Gutenkunst et al. 2009), which lacks polarization of SNPs (Fig. S1).

185 Because we had prior evidence that these species represent two genetic populations

186 (based on taxonomy and results from Maley \& Winker 2010), we used $\delta$ a $\delta i$ to infer what general 187 two-population divergence model best fit the data. We then used that model to estimate 
188 demographic parameters (i.e., effective population sizes, split time, and migration). We ran six

189 different models spanning the standard possible demographic histories of two populations, five

190 basic and one derivative: 1) neutral (no divergence, or still strongly mixing), 2) split with

191 migration, 3) split with no migration, 4) isolation with bidirectional migration and population

192 growth, 5) isolation with population growth and no migration, and 6) a custom split-

193 bidirectional-migration model (a simple derivative of split-migration; Fig. 1). The neutral, split-

194 with-migration, and isolation-with-migration-and-population-growth models are provided in the

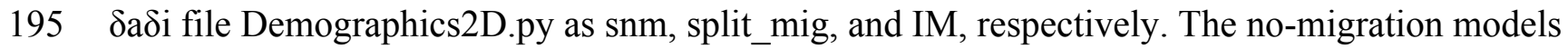

196 (3 and 5 above) use the split_mig and IM models, respectively, with migration parameters set to

197 zero. The split-bidirectional-migration model (figshare

198 https://doi.org/10.6084/m9.figshare.6453125.v1) adds bidirectional migration to the split-

199 migration model to examine potential asymmetry in gene flow.

200 We performed a series of optimization runs (8-47 each) of each basic model, adjusting

201 parameters (grid points, upper and lower bounds) to identify high log composite likelihoods. We

202 then ran each model repeatedly, varying parameters within bounds that yielded the highest

203 likelihood during optimization, until three runs yielded the highest observed likelihood value.

204 Our reasoning was that this level of repeatability indicated a best-fit neighborhood for each

205 model. We report this highest observed likelihood, except for poorer models, which yielded

206 variable likelihoods, in which case we averaged and report the highest five values. After

207 identifying the best-fit model based on likelihood values over successive runs, we ran the best-fit

208 model ten times each with jackknifed datasets to estimate the $95 \%$ confidence interval (CI) for

209 each parameter.

210 We estimated the average per-site substitution rate by BLASTing the FASTA file

211 containing all confidently scored loci (those meeting our quality filters as described in the

212 Bioinformatics section, above) for all individuals (3,431 loci) against the budgerigar

213 (Melopsittacus undulatus) genome (NCBI release 102) and the rifleman (Acanthisitta chloris)

214 genome (NCBI release 100), using time to most recent common ancestor (TMRCA) date

215 estimates of 60.5 Ma (budgerigar) and $53 \mathrm{Ma}$ (rifleman) (Claramunt \& Cracraft 2015). These

216 taxa were chosen as the nearest relatives with complete genomes and fossil-dated nodes

217 available. We imported BLAST results (as a hit table, csv) into a spreadsheet, removed duplicate

218 lower-affinity hits, then summed total length of base pairs, total substitutions, and calculated 
219 substitutions per site. This value (substitutions per site) was annualized by multiplying it by 2

220 TMRCA (e.g., 121 Ma for the total time along the branches of divergence between buntings and

221 budgerigars). To account for the uncertainty associated with using divergence time estimates

222 from distant relatives, we averaged the resulting substitutions per site per year rates $\left(6.83 \times 10^{-10}\right.$

223 and $6.67 \times 10^{-10}$, respectively), and we used the average rate $\left(6.75 \times 10^{-10}\right)$ to convert parameter

224 estimates obtained from $\delta a \delta i$ analyses into biologically relevant estimates of effective population

225 size(s) and split time(s). We converted substitution rates to substitutions/site/generation using a

226 generation time of $2.7 \mathrm{yr}$ for snow buntings. We estimated generation time using survival and

227 breeding data from Smith (1994) and the method of Saether et al. (2005), in which generation

228 time $(G)$ is calculated as $G=\alpha+(s /(1-s))$, where $\alpha$ is age of first breeding (1 yr) and $s$ is annual

229 adult survival. Finally, because our $\delta a \delta i$ analyses used filtered SNPs, our demographic estimates

230 used an adjusted surveyed sequence length of: (total sites surveyed, including invariant

231 sites)*(proportion of SNPs used after thinning to $1 \mathrm{SNP} /$ locus $)=1,103,715 \mathrm{bp}$.

232

233 Results

234 Assembly produced 632,401 contigs $(\min =224 \mathrm{bp}, \max =17,453 \mathrm{bp})$ with a mean

235 length of $396.6 \mathrm{bp}( \pm 0.27 \mathrm{bp} 95 \% \mathrm{CI})$ for a total of $250,802,355 \mathrm{bp}$. Fully 9,194 contigs were

236 over $1 \mathrm{~Kb}$ in length. After identifying UCE loci and removing potential paralogs, we recovered

237 4,018 UCE loci. After filtering UCE loci for quality, calling SNPs, phasing (reconstructing

238 haplotypes), and applying additional quality filters, we identified 2,635 loci that contained data

239 for all individuals and were variable. This complete matrix of variable loci included a total of

240 9,449 SNPs (averaging 3.6 sites per locus). Per-site sequencing depth for these SNPs averaged

24126.3 reads $( \pm$ 16.9 SD). An additional 587 loci exhibited variation but the data were not of

242 sufficient quality (i.e., GQ < 10) among all individuals to confidently call both alleles. There

243 were 796 high-quality invariant loci (loci with invariant data, rather than an absence of data),

244 providing a full dataset of 3,431 loci with mean length of $1,153.6 \mathrm{bp}( \pm 4.95 \mathrm{bp} 95 \% \mathrm{CI})$. The

245 shortest locus was $228 \mathrm{bp}$, the longest 2,543 bp, and 2,482 loci were longer than $1 \mathrm{~Kb}$ (Fig. S2).

246 The total length of these loci was 3,957,876 bp. The distribution of SNP variation among loci

247 confidently called for all individuals is given in Fig. 2. Nucleotide diversity $(\pi)$ was 0.000519

248 overall, 0.000523 for snow buntings, and 0.000493 for McKay’s buntings. 
No alleles showed fixed differences $\left(F_{S T}=1.0\right)$ between the two populations, and few

250 alleles showed strong segregation. No variable sites had an $F_{S T}$ value above 0.9 , and there were

251 only three each at 0.86 and 0.72 (Fig. S3; two of these sites were on the same locus). One of the

252 five loci with the highest $F_{S T}$ values was Z-linked; all of the others were on different

253 chromosomes (figshare https://doi.org/10.6084/m9.figshare.6453125.v1). There were $128 \mathrm{Z}$ -

254 linked loci among the 2,635 variable loci. As noted, only one showed high $F_{S T}$ between the two

255 species. The two populations had an overall $F_{S T}=0.034$, which was significant $(P=0.03)$. The

256 average distance between taxa $\left(d_{\mathrm{xy}}\right)$ was $5.3 \times 10^{-4}$, and the net average distance $\left(d_{\mathrm{A}}\right)$ was $2.0 \times$

257 10-5. Discriminant Analysis of Principal Components (DAPC in adegenet) assigned all

258 individuals to their correct taxon of origin (retaining the first $4 \mathrm{PCs}$ ), with $100 \%$ probabilities for 259 each, indicating a high level of genomic diagnosability (Fig. S4).

260 Fully 2,510 loci were in Hardy-Weinberg equilibrium; 124 were not (one was triallelic).

261 McKay's buntings had fewer unique alleles $(4,238)$ than snow buntings $(4,389)$, concordant with

262 the smaller population size of McKay's buntings. Bartlett's test rejected homogeneity of

263 variance between observed heterozygosity $\left(H_{o}=0.18,0.19\right)$ and expected heterozygosity $\left(H_{e}=\right.$

$2640.20,0.22)$, but $H_{o}$ did not differ from $H_{e}(t=-3.1653, \mathrm{df}=2633, P=1.0)$.

265

266

The four-gametes test suggested that recombination occurred in hundreds of loci. For 405

267

loci, locus lengths were shortened by IMgc to meet the four-gametes test, and for 252 loci one or more individuals were removed to meet the same criteria (a few of these loci had both done;

IMgc automatically performs one or the other or both operations to obtain non-recombinant sequence data). There were thus $15.4 \%$ to $24.9 \%$ of variable loci exhibiting patterns indicative of recombination. As noted in the Methods, these sequence data, together with all other unchanged sequences, were not used further; we used only SNP data for further analyses.

In testing our six, two-population models with $\delta a \delta i$, the highest maximum log composite 273 likelihood values were obtained for the split-with-migration model (-112.76), which made it the 274 best-fitting model for these data (model 2 in Fig. 1). We obtained successively lower likelihood 275 values for the neutral (-588.45), isolation with bidirectional migration and population growth (276 803.30), and isolation with population growth and no migration $(-2,026.93)$ models. The final

277 model tested, split-bidirectional-migration, had an intermediate likelihood of -286.49 . The split278 with-no-migration model was unstable under all conditions tried, and we could not get it to run 
279 to convergence. We provide jackknifed estimates and confidence intervals for the best-fitting, 280 split-with-migration model in Table 1.

281

\section{Discussion}

283 Our data provided sufficient variation to answer fundamental questions about these two 284 recently diverged taxa, despite a lack of fixed genetic differences and evidence for moderate 285 levels of gene flow. Thus, our study adds to evidence showing the utility of UCEs for 286 illuminating key evolutionary attributes among populations with shallow levels of divergence 287 (e.g., Table 2). These data also provide a direct comparison to markers previously used to 288 investigate recent divergence (i.e., mtDNA, AFLPs) for these same taxa (see below). As UCEs 289 are used more frequently for population genomics, in addition to systematics, new actions 290 become desirable (Table 2). Some of the key approaches are: sequencing at increased depth, 291 genotyping individuals (determining both alleles of a locus), implementing genotype quality 292 filters, accounting for recombination, improving mutation rate estimates, and implementing 293 population genomics analytical pipelines rather than those oriented more typically toward systematics. Questions often differ at population levels, but researchers are successfully applying a variety of approaches that demonstrate the utility of UCEs in population genomics (Table 2).

In considering UCEs as a class of markers that subsamples the genome, it is useful to note that our estimated substitution rates (mean of $6.75 \times 10^{-10}$ substitutions per site per year) are roughly an order of magnitude slower than the mutation rate estimated across the entire genome of three generations of Ficedula flycatchers (Smeds et al. 2016). This is perhaps not surprising given the conserved nature of these loci. Using a very different method to estimate substitution rates (scaling UCE results to an mtDNA molecular clock), Harvey et al. (2016) estimated rates of $1.74 \times 10^{-12}$ to $2.32 \times 10^{-11}$ substitutions per site per year for UCE loci, one to two orders of magnitude slower than comparable RAD-seq data from the same animals and also slower than

304 the rates we estimated here for buntings. In addition to differences in methodology, Harvey et al.

305 (2016) had shorter loci on average (mean locus length $604 \mathrm{bp}$ ) than loci in our study.

306 Nevertheless, more study of substitution rates in loci with UCEs is warranted because these

307 estimates are important when converting modeled demographic parameters into biological units.

308 The effect of our estimated substitution rates on our demographic estimates (if, for example, our 309 substitution rate estimates are wrong) is that for some, they are positively correlated; lower 
310 substitution rates would drive effective population sizes and split times lower $\left(N_{e}: n u 1, n u 2\right.$, and

311 Nref and $T$ in Table 1). Migration rate $(m)$ estimates in Table 1 are unaffected by substitution

312 rates.

313 The nucleotide diversity levels that we observed are approximately an order of magnitude

314 lower than typical levels across the avian genome (0.0011- 0.005; Ellegren 2013). This is likely

315 the result of purifying selection acting on UCE loci, effecting an apparent lower substitution rate.

316 Our values are more similar to the values for Z-linked loci in other bird species (e.g.,

317 Balakrishnan \& Edwards 2009, Huynh et al. 2010, Lavretsky et al. 2015).

318 When applied to this relatively recently derived pair of taxa, UCE results raise the

319 question of whether McKay's bunting is a full biological species. Although McKay's bunting is

320 taxonomically recognized as a species, this dataset shows substantial levels of gene flow (see

321 Wright 1943, Cabe and Alstad 1994, Winker 2010), and the lack of fixed alleles is surprising

322 given that we sampled thousands of loci and four individuals from each of two putative species;

323 we would expect several fixed differences to occur by chance through neutral processes. There

324 are some noted plumage differences between the two taxa (Maley \& Winker 2007). But while

325 our results enabled 100\% diagnosability (which might decline with broader sampling; it was

326 96.5\% using AFLPs in Maley \& Winker 2010), they also suggest widespread genomic similarity

327 between McKay's and snow buntings (e.g., relatively low $F_{S T}$ ). Given phenotypic differences

328 between the taxa, it seems likely that there are fixed allelic differences in portions of the genome

329 not included in our data that could be detected by more extensive surveys of each species'

330 genome. The status of the taxa as biological species, however, is more likely to hinge on gene

331 flow (i.e., the geographic partitioning of traits that may be responding to adaptation is not

332 equivalent to speciation).

333 There are reports of male McKay's buntings present outside their breeding range and

334 possible hybridization between McKay's and snow buntings (Sealy 1967, 1969). Snow buntings

335 are also common on the breeding range of McKay's buntings at St. Matthew Island prior to and

336 during early portions of the breeding season, although most individuals leave before fledging

337 (Winker et al. 2002). Just one pair of snow buntings has been recorded on the island during

338 fledging (Winker et al. 2002). Observations thus suggest the possibility of hybridization; our data

339 provide a confirmation and a quantification of it. The levels of gene flow that we found, $2.8-5.2$

340 individuals per generation (Table 1), seem rather high for two putative biological species (Rice 
341 \& Hostert 1993, Hostert 1997, Winker 2010). Further study will be needed to determine species

342 limits between these taxa, including larger sample sizes, broader genomic coverage, and proper

343 caution for interpreting genomic results in terms of species delimitation (Robinson et al. 2013,

344 Sukumaran \& Knowles 2017).

345 In comparing UCE-based estimates of demographic parameters with those based on

346 mtDNA sequence (Maley \& Winker 2010), we find little overlap (Table 3), and our UCE-based

347 split-time estimate is an order of magnitude earlier. Although effective population size estimates

348 for McKay's buntings are close (though non-overlapping), those for snow buntings are one-to-

349 two orders of magnitude smaller, a difference that is only partially explained by differences in

350 effective population size for autosomal and mtDNA estimates. These differences may also be

351 driven by the different selection regimes operating on the two marker classes. For example,

352 purifying selection on UCEs will result in background selection on linked variation in flanking

353 regions, reducing (through hitchhiking) the effective population size (Charlesworth \&

354 Charlesworth 2016). Previously, mtDNA results suggested that gene flow was highly

355 asymmetric (Maley \& Winker 2010), concordant with what was likely a post-glacial

356 introgression of McKay's buntings into snow buntings during a snow bunting range expansion

357 into Beringia. Our UCE-based estimates have much narrower confidence limits (and without the

358 strong asymmetry found in mtDNA; Table 3), but they do suggest moderate levels of gene flow

359 between the two species.

360

\section{Conclusions}

362 Although more work is needed to understand demographic estimates made using UCEs

363 relative to those obtained using other markers, UCEs provide rich, high-quality data for

364 population genomic studies (Table 4). They are thus an important new class of genomic marker

365 that should provide broad comparative value among diverse population genomics studies, with

366 ever-increasing value as additional studies using UCEs (or whole genomes from which UCEs

367 can be obtained) are conducted.

368

369 Acknowledgments

370 Oralee Nudson provided invaluable guidance for supercomputer use. Matthew Miller, Mike

371 Harvey, and Naoki Takebayashi provided helpful suggestions in bioinformatics, and Jessica 
372 McLaughlin provided a script for Fig. S2. Kevin Hawkins, Phil Lavretsky, Jeff Peters, and Ryan

373 Gutenkunst provided help in running dadi. We also thank Iris Cato, Maryanne Evans, Katie

374 Everson, Angela Gastaldi, Jessica McLaughlin, Kendall Mills, Katie Shink, Naoki Takebayashi,

375 and Sara Wilbur for helpful comments.

376

377

378

379

380

381

382

383

384

385

386

387

388

389

390

391

392

393

394

395

396

397

398

399

400

401

402

403

404

405

406

407

408

409

410

411

412

413

414

415

\section{Literature Cited}

Afgan E, Baker D, van den Beek M, Blankenberg D, Bouvier D, Cech M, Chilton J, Clements D, Coraor N, Eberhard C, Grüning B, Guerler A, Hillman-Jackson J, Von Kuster G, Rasche E, Soranzo N, Turaga N, Taylor J, Nekrutenko A, Goecks J. 2016. The Galaxy platform for accessible, reproducible and collaborative biomedical analyses: 2016 update. Nucleic Acids Research 44:W3-W10. doi: 10.1093/nar/gkw343

Avise JC. 1994. Molecular Markers, Natural History, and Evolution. Chapman \& Hall, New York.

Balakrishnan CN, Edwards SV. 2009. Nucleotide variation, linkage disequilibrium and founderfacilitated speciation in wild populations of the zebra finch (Taeniopygia guttata). Genetics 181:645-60.

Bolger AM, Lohse M, Usadel B. 2014. Trimmomatic: A flexible trimmer for Illumina Sequence Data. Bioinformatics 30:2114-2120. http://dx.doi.org/10.1093/bioinformatics/btu170.

Cabe PR, Alstad DN. 1994. Interpreting population differentiation in terms of drift and selection. Evolutionary Ecology 8:489-492.

Charlesworth B, Charlesworth D. 2016. Population genetics from 1966 to 2016. Heredity 2016:1-8.

Danecek P, Auton A, Abecasis G, Albers CA, Banks E, DePristo MA, Handsaker RE, Lunter G, Marth GT, Sherry ST, McVean G, Durbin R, 1000 Genomes Project Analysis Group. 2011. The Variant Call Format and VCFtools. Bioinformatics 27:2156-2158.

Ellegren H. 2013. The evolutionary genomics of birds. Annual Review of Ecology Evolution \& Systematics 44:239-59.

Ellegren H. 2014. Genome sequencing and population genomics in non-model organisms. Trends in Ecology \& Evolution 29:51-63.

Faircloth BC. 2013. illumiprocessor: a trimmomatic wrapper for parallel adapter and quality trimming. http://dx.doi.org/10.6079/J9ILL.

Faircloth BC 2016. PHYLUCE is a software package for the analysis of conserved genomic loci. Bioinformatics 32:786-788. doi: 10.1093/bioinformatics/btv646. 
416

417

418

419

420

421

422

423

424

425

426

427

428

429

430

431

432

433

434

435

436

437

438

439

440

441

442

443

444

445

446

447

448

449

450

451

452

453

454

455

456

457

458

459

460

461
Faircloth BC, McCormack JE, Crawford NG, Harvey MG, Brumfield RT, Glenn TC. 2012. Ultraconserved elements anchor thousands of genetic markers for target enrichment spanning multiple evolutionary timescales. Systematic Biology 61:717-726. doi:10.1093/sysbio/SYS004

Faircloth BC, Branstetter MG, White ND, Brady SG. 2015. Target enrichment of ultraconserved elements from arthropods provides a genomic perspective on relationships among Hymenoptera. Molecular Ecology Research 15:489-501. doi:10.1111/1755-0998.12328

Felsenstein J. 2005. Accuracy of coalescent likelihood estimates: Do we need more sites, more sequences, or more loci? Molecular Biology and Evolution 23:691-700.

Garrigan D, Kingan SB, Pilkington MM, Wilder JA, Cox MP, Soodyall H, Strassmann B, Destro-Bisol G, de Knijff P, Novelletto A, Friedlaender J, Hammer MF. 2007. Inferring human population sizes, divergence times and rates of gene flow from mitochondrial, $\mathrm{X}$ and Y chromosome resequencing data. Genetics 177:2195-2207.

Gilbert PS, Chang J, Pan C, Sobel E, Sinsheimer JS, Faircloth BC, Alfaro ME. 2015. Genomewide ultraconserved elements exhibit higher phylogenetic informativeness than traditional gene markers for the fish series Percomorpha. Molecular Phylogenetics \& Evolution 92:140-146. doi:10.1016/j.ympev.2015.05.027

Glenn TC, Nilsen R, Kieran TJ, Finger Jr JW, Pierson TW, Bentley KE, Hoffberg SL, Louha S, García-De-Leon FJ, Portilla MAR, Reed K, Anderson JL, Meece JK, Aggrey S, Rekaya R, Alabady M, Belanger M, Winker K, Faircloth BC. 2017. Adapterama I: Universal stubs and primers for thousands of dual-indexed Illumina libraries (iTru \& iNext). Molecular Ecology Resources (accepted), available at http://biorxiv.org/content/early/2016/06/15/049114

Goudet J, Raymond M, de Meeüs T, Rousset F. 1996. Testing differentiation in diploid populations. Genetics 144:1933-1940.

Grabherr MG, Haas BJ, Yassour M, Levin JZ, Thompson DA, Amit I, Adiconis X, Fan L, Raychowdhury R, Zeng Q, Chen Z, Mauceli E, Hacohen N, Gnirke A, Rhind N, di Palma F, Birren BW, Nusbaum C, Lindblad-Toh K, Friedman N, Regev A. 2013. Trinity: reconstructing a full-length transcriptome without a genome from RNA-Seq data. Nature Biotechnology 29:644-652.

Gutenkunst RN, Hernandez RD, Williamson SH, Bustamante CD. 2009. Inferring the joint demographic history of multiple populations from multidimensional SNP data. PLoS Genetics 5:e1000695.

Harvey MG, Brumfield RT. 2015. Genomic variation in a widespread Neotropical bird (Xenops minutus) reveals divergence, population expansion, and gene flow. Molecular Phylogenetics \& Evolution 83:305-316. 
462 Harvey MG, Smith BT, Glenn TC, Faircloth BC, Brumfield RT. 2016. Sequence capture versus

463

464

465

466

467

468

469

470

471

472

473

474

475

476

477

478

479

480

481

482

483

484

485

486

487

488

489

490

491

492

493

494

495

496

497

498

499

500

501

502

503

504

505

506

507

508 restriction site associated DNA sequencing for shallow systematics. Systematic Biology 65:910-924.

Harvey MG, Aleixo A, Ribas CC, Brumfield RT. 2016. Habitat preference predicts genetic diversity and population divergence in Amazonian birds. American Naturalist 190:631-648.

Hillis DM, Moritz C, Mable BK (eds). 1996. Molecular Systematics, $2^{\text {nd }}$ ed. Sinauer Associates, Inc. Sunderland, Massachusetts.

Hostert EE. 1997. Reinforcement: A new perspective on an old controversy. Evolution 51:697702.

Huynh LY, Maney DL, Thomas JW. 2010. Contrasting population genetic patterns within the white-throated sparrow genome (Zonotrichia albicollis). BMC Genetics 11:96.

Jombart T, Ahmed I. 2011. adegenet 1.3-1: new tools for the analysis of genome-wide SNP data. Bioinformatics 27:3070-3071. doi:10.1093/bioinformatics/btr521

Jorde LB, Watkins WS, Bamshad MJ, Dixon ME, Ricker CE, Seielstad MT, Batzer MA. 2000. The distribution of human genetic diversity: A comparison of mitochondrial, autosomal, and Y-chromosome data. American Journal of Human Genetics 66:979-988.

Lavretsky P, Peters JL, Winker K, Bahn V, Kulikova I, Zhuravlev YN, Wilson RE, Barger C, Gurney K, McCracken KG. 2016. Becoming pure: identifying generational classes of admixed individuals within lesser an greater scaup populations. Molecular Ecology 25:661-674.

Leaché AD, Chavez AS, Jones LN, Grummer JA, Gottscho AD, Linkem CW 2015. Phylogenomics of Phrynosomatid lizards: Comflicting signals from sequence capture versus restriction site associated DNA sequencing. Genome Biology and Evolution 7:706-719.

Li H. 2013. Aligning sequence reads, clone sequences and assembly contigs with BWA-MEM. https://arxiv.org/abs/1303.3997

Li H, Durbin R. 2010. Fast and accurate long-read alignment with Burrows-Wheeler transform. Bioinformatics 26:89-595.

Li H, Handsaker B, Wysoker A, Fennell T, Ruan J, Homer N, Marth G, Abecasis G, Durbin R, 1000 Genome Project Data Processing Subgroup. 2009. The sequence alignment/map format and SAMtools. Bioinformatics 25:2078-2079.

Lischer HEL, Excoffier L. 2012. PGDSpider: An automated data conversion tool for connecting population genetics and genomics programs. Bioinformatics 28:298-299.

Maley JM, Winker K. 2007. The utility of juvenal plumage in diagnosing species limits: An example using buntings in the genus Plectrophenax. Auk 124:907-915.

Peer] reviewing PDF | (2018:03:27201:2:0:NEW 14 Aug 2018) 
509

510

511

512

513

514

515

516

517

518

519

520

521

522

523

524

525

526

527

528

529

530

531

532

533

534

535

536

537

538

539

540

541

542

543

544

545

546

547

548

549

550

551

552

553

554

Maley JM, Winker K. 2010. Diversification at high latitudes: Speciation of buntings in the genus Plectrophenax inferred from mitochondrial and nuclear markers. Molecular Ecology 19:785-797.

Manthey JD, Campillo LC, Burns KJ, Moyle RG. 2016. Comparison of target-capture and restriction-site associated DNA sequencing for phylogenomics: A test in cardinalid tanagers (Aves, genus: Piranga). Systematic Biology 65:640-650.

Marcovitz A, Jia R, Bejerano G. 2016. "Reverse genomics" predicts function of human conserved noncoding elements. Molecular Biology \& Evolution 33:1358-1369.

Mason NA, Olvera-Vital A, Lovette IJ, Navarro-Sigüenza AG. 2018. Hidden endemism, deep polyphyly, and repeated dispersal across the Isthmus of Tehuantepec: Diversification of the White-collared Seedeater complex (Tharupide: Sporophila torqueola). Ecology and Evolution 8:1867-1881.

McCormack JE, Harvey MG, Faircloth BC, Crawford NG, Glenn TC, Brumfield RT. 2013. A phylogeny of birds based on over 1,500 loci collected by target enrichment and highthroughput sequencing. PLoS ONE 8:e54848. doi:10.1371/journal.pone.0054848

McKenna A, Hanna M, Banks E, Sivachenko A, Cibulskis K, Kernytsky A, Garimella K, Altshuler D, Gabriel S, Daly M, DePristo MA. 2010. The Genome Analysis Toolkit: a MapReduce framework for analyzing next-generation DNA sequencing data. Genome Research 20:1297-303.

Milne I, Stephen G, Bayer M, Cock PJA, Pritchard L, Cardle L, Shaw PD, Marshall D. 2013. Using Tablet for visual exploration of second-generation sequencing data. Briefings in Bioinformatics 14:193-202.

Oswald JA, Harvey MG, Remsen RC, Foxworth DU, Cardiff SW, Dittmann DL, Megna LC, Carling MD, Brumfield RT. 2016. Willet be one species or two? A genomic view of the evolutionary history of Tringa semipalmata. Auk 133:593-614.

Pearse DE, Crandall KA. 2004. Beyond $\mathrm{F}_{\mathrm{ST}}$ : Analysis of population genetic data for conservation. Conservation Genetics 5:585-602.

Rohland N, Reich D. 2012. Cost-effective, high-throughput DNA sequencing libraries for multiplexed target capture. Genome Research 22:939-946.

Rice WR, Hostert EE. 1993. Laboratory experiments on speciation: What have we learned in 40 years? Evolution 47:1637-1653.

Robinson, J. D., A. C. Coffman, M. J. Hickerson, and R. N. Gutenkunst. 2014. Sampling strategies for frequency spectrum-based population genomic inference. BMC Evolutionary Biology 14:254. 
555

556

557

558

559

560

561

562

563

564

565

566

567

568

569

570

571

572

573

574

575

576

577

578

579

580

581

582

583

584

585

586

587

588

589

590

591

592

593

594

595

596

597

598

Sæther B-E, Lande R, Engen S, Weimerskirsch H, Lillegård M, Altwegg R, Becker PH, Bregnballe J, Brommer JW, McCleery RH, Merilä J, Nyholm E, Rendell W, Robertson RR, Tryjanowski P, Visser ME. 2005. Generation time and temporal scaling of bird population dynamics. Nature 436:99-102.

Sethuraman A, Hey J. 2015. IMa2p-parallel MCMC and inference of ancient demography under the Isolation with migration (IM) model. Molecular Ecology Resources 16:206-215. DOI: http://dx.doi.org/10.1111/1755-0998.12437

Sealy SG. 1967. The occurrence and possible breeding of McKay's bunting on St. Lawrence Island, Alaska. Condor 69:531-532.

Sealy SG. 1969. Apparent hybridization between snow bunting and McKay's bunting on St. Lawrence Island, Alaska. Auk 86:350-351.

Smeds L, Qvarnström A, Ellegren H. 2016 Direct estimate of the rate of germline mutation in a bird. Genome Research 26:1211-1218.

Smith RD. 1994. Snow buntings Plectrophenax nivalis: the behavioural ecology and site use of an itinerant flock species in the nonbreeding season. $\mathrm{PhD}$ thesis, University of Glasgow.

Smith BT, Harvey MG, Faircloth BC, Glenn TC, Brumfield RT. 2014. Target capture and massively parallel sequencing of ultraconserved elements (UCEs) for comparative studies at shallow evolutionary time scales. Systematic Biology 63: 83-95. doi:10.1093/sysbio/syt061

Sukumaran J, Knowles LL. 2017. Multispecies coalescent delimits structure, not species. Proceedings of the National Academy of Sciences 114: 1607-12.

Tamura K, Stecher G, Peterson D, Filipski A, Kumar S. 2013. MEGA6: Molecular Evolutionary Genetics Analysis version 6.0. Molecular Biology and Evolution 30:2725-2729.

Winker K. 2010. Subspecies represent geographically partitioned variation, a goldmine of evolutionary biology, and a challenge for conservation. Ornithological Monographs 67:623.

Winker K, Gibson DD, Sowls AL, Lawhead BE, Martin PD, Hoberg EP, Causey D. 2002. The birds of St. Matthew Island. Wilson Bulletin 114:491-509.

Woerner AE, Cox MP, Hammer MF. 2007. Recombination-filtered genomic datasets by information maximization. Bioinformatics 23:1851-1853.

Wright S. 1943. Isolation by distance. Genetics 28:114-138. 
599 Zarza E, Faircloth BC, Tsai WLE, Bryson Jr. RW, Klicka J, McCormack JE. 2016. Hidden 600 histories of gene flow in highland birds revealed with genomic markers. Molecular Ecology $601 \quad 25: 5144-5157$.

602

603 Zhang Z, Schwartz S, Wagner L, Miller W. 2000. A greedy algorithm for aligning DNA 604 sequences. Journal of Computational Biology 7:203-14.

605 


\section{Figure 1 (on next page)}

Population divergence models tested using $\delta a \delta i$

Population divergence models tested using $\delta a \delta i$, varying from 1 ) neutral (no divergence), to a series of different two-population models with an ancestral population diverging into two populations (at time $T$ ): 2) split with migration (gene flow), 3) split with no migration, 4) isolation with bidirectional migration and population growth, 5) isolation with population growth and no migration, and 6) a derivative of model 2 with bidirectional migration. 


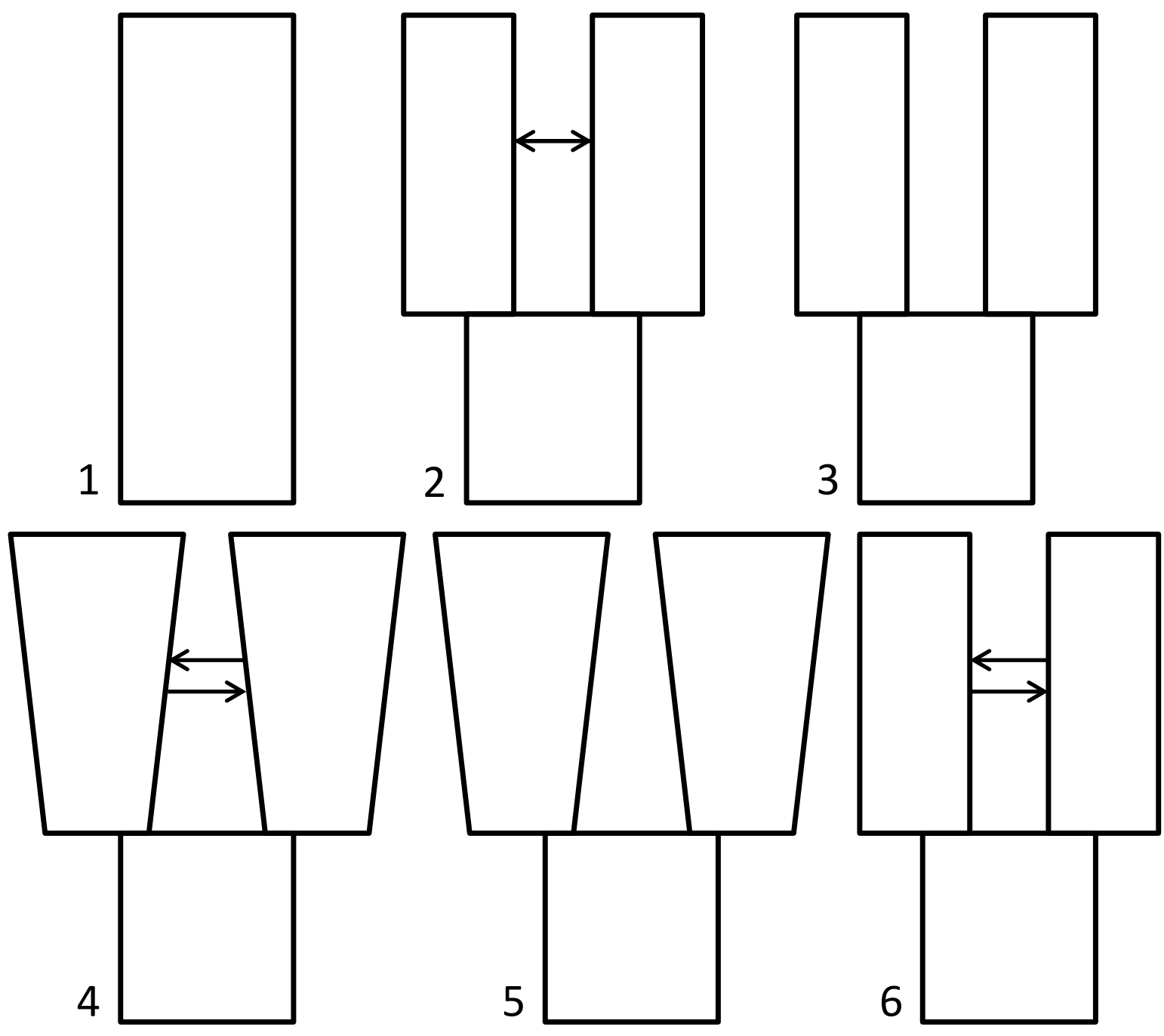




\section{Figure 2 (on next page)}

Distribution of single nucleotide polymorphisms (SNPs) per locus

Distribution of single nucleotide polymorphisms (SNPs) per locus among 3,431 confidently called loci. 


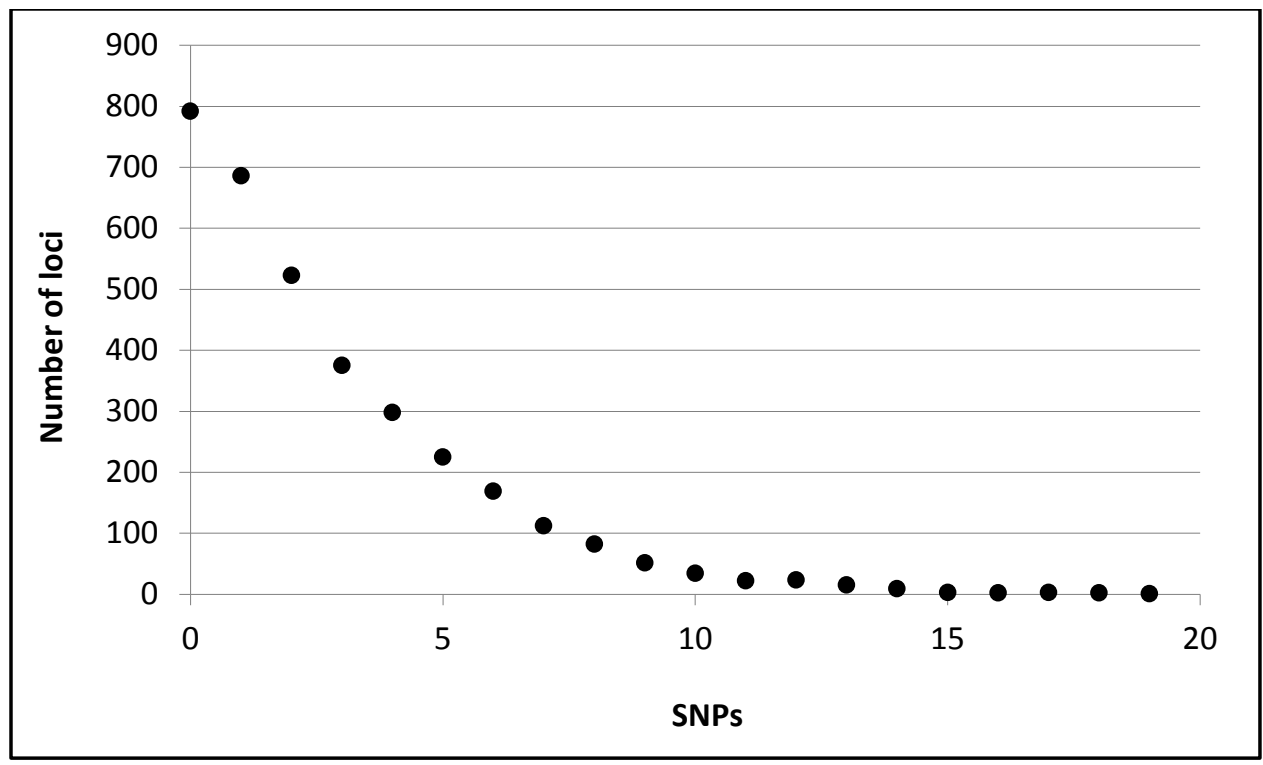




\section{Table $\mathbf{1}$ (on next page)}

Demographic model parameters

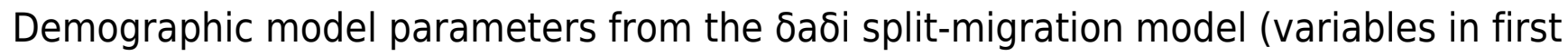
column) and estimates in biological units (defined in final column), with $95 \% \mathrm{Cls}$ determined by jackknifed datasets. The two migration rates use the two different effective population sizes in their calculation. 
1 Table 1. Demographic model parameters from the $\delta a \delta i$ split-migration model (variables in first 2 column) and estimates in biological units (defined in final column), with $95 \%$ Cls determined by 3 jackknifed datasets. The two migration rates use the two different effective population sizes in 4 their calculation.

5

\begin{tabular}{|c|c|c|c|c|}
\hline Model parameters & $\begin{array}{c}\text { Parameter (+ } \\
95 \% \mathrm{Cl})\end{array}$ & $\begin{array}{c}\text { Estimates (+ 95\% } \\
\mathrm{Cl})\end{array}$ & $\begin{array}{l}\text { Lower-upper } \\
\text { bounds }\end{array}$ & Biological units \\
\hline nu1 (pop size & & $109,330( \pm$ & & \\
\hline McKay's) & $3.52( \pm 0.54)$ & $16,790)$ & $92,540-126,120$ & individuals McKay's \\
\hline nu2 (pop size & & $184,991( \pm$ & & \\
\hline snow) & $5.95( \pm 1.79)$ & $55,523)$ & $129,467-240,514$ & individuals snow \\
\hline$T$ (split time) & $1.44( \pm 0.37)$ & $\begin{array}{c}241,491( \pm \\
62,429)\end{array}$ & $179,061-303,920$ & years \\
\hline$m_{1}$ (migration) & $1.65( \pm 0.39)$ & $2.90( \pm 0.10)$ & $2.8-3.0$ & individuals using nu1 \\
\hline$m_{2}$ (migration) & $1.65( \pm 0.39)$ & $4.90( \pm 0.35)$ & $4.6-5.2$ & individuals using nu2 \\
\hline theta & $\begin{array}{c}249.97( \pm \\
32.71)^{\mathrm{a}}\end{array}$ & $31,072( \pm 4,066)^{a}$ & $27,006-35,138$ & $\begin{array}{l}\text { ancestral population } \\
\text { individuals }\end{array}$ \\
\hline
\end{tabular}

a - Nref 


\section{Table 2 (on next page)}

Some bioinformatic and analytical attributes typical of population genomics studies using UCEs.

Some bioinformatic and analytical attributes typical of population genomics studies and some of the variation among researchers in applying them to different questions using UCE data. 
1 Table 2. Some bioinformatic and analytical attributes typical of population genomics studies

2 and some of the variation among researchers in applying them to different questions using UCE 3 data.

4

5

\begin{tabular}{|c|c|c|c|c|c|}
\hline Popgen attribute & $\begin{array}{l}\text { Smith et al. } \\
2014\end{array}$ & $\begin{array}{l}\text { Harvey et al. } \\
2016\end{array}$ & $\begin{array}{l}\text { Zarza et al. } \\
2016\end{array}$ & $\begin{array}{l}\text { Oswald et al. } \\
2016\end{array}$ & $\begin{array}{l}\text { this } \\
\text { study }\end{array}$ \\
\hline Genotyping & no & yes & yes & yes & yes \\
\hline GQ filters & no & yes & yes & ? & yes \\
\hline recombination & no & no & no & no & yes \\
\hline $\begin{array}{l}\text { substitution rates } \\
\text { population }\end{array}$ & yes & yes & no & yes & yes \\
\hline differentiation & yes & yes & yes & yes & yes \\
\hline $\begin{array}{l}\text { gene flow rates } \\
\text { effective pop. }\end{array}$ & yes & no & no & yes & yes \\
\hline sizes & yes & yes & no & yes & yes \\
\hline heterozygosity & no & yes & no & no & yes \\
\hline
\end{tabular}

6

7

8

9

10 


\section{Table 3(on next page)}

Comparing UCE and mtDNA demographic estimates

Comparing bunting UCE results of demographic estimates with those obtained using mtDNA by Maley \& Winker (2010). 
1 Table 3. Comparing bunting UCE results of demographic estimates with those obtained using 2 mtDNA by Maley \& Winker (2010).

3

\begin{tabular}{lcc} 
Parameter & UCEs & mtDNA \\
\hline split time & $179-304 \mathrm{Kyr}$ & $18-74 \mathrm{Kyr}$ \\
$\boldsymbol{N}_{e}$ McKay's & $93-126 \mathrm{~K}$ & $170-680 \mathrm{~K}$ \\
$\boldsymbol{N}_{e}$ snow & $0.13-0.24$ million & $6-24$ million \\
$\boldsymbol{m}$ & $2.8-5.2$ & $0.05-753$ \\
\hline
\end{tabular}

4

5

6 
Table 4(on next page)

Comparing avian UCE population genomic characteristics. 
1 Table 4. Comparing avian UCE population genomic characteristics.

2

\begin{tabular}{|c|c|c|c|c|c|}
\hline Species & \# loci & $\begin{array}{l}\% \\
\text { polymorphic }\end{array}$ & $\begin{array}{l}\text { Nucleotide } \\
\text { diversity }\end{array}$ & Heterozygosity & Source \\
\hline Tringa semipalmata & 4635 & 94 & n.a. & n.a. & Oswald et al. 2016 \\
\hline Cymbilaimus lineatus & 776 & 53 & 0.0019 & n.a. & Smith et al. 2014 \\
\hline Xenops minutus & 1368 & 73 & 0.0019 & n.a. & Smith et al. 2014 \\
\hline Schiffornis turdina & 851 & 77 & 0.0003 & n.a. & Smith et al. 2014 \\
\hline Querula purpurata & 1516 & 58 & 0.0013 & n.a. & Smith et al. 2014 \\
\hline Microcerculus marginatus & 1077 & 60 & 0.0015 & n.a. & Smith et al. 2014 \\
\hline $\begin{array}{l}\text { Plectrophenax spp. (2) } \\
\text { average of } 40 \text { Amazonian }\end{array}$ & 3431 & 77 & 0.0005 & $0.20-0.22$ & this study \\
\hline species & 2416 & varied & 0.0011 & 0.44 (1 sp.) & Harvey et al. 2016 \\
\hline
\end{tabular}

3 